\title{
The Clinical Features and Survival Outcome of 107 Newly Diagnosed Advanced Stage Extranodal NK/ T-Cell Lymphoma Cases: A Triple-Center Study
}

This article was published in the following Dove Press journal: Cancer Management and Research

\author{
Jiwei $\operatorname{Li} \mathbb{D}^{1-3}$ \\ Jin $\mathrm{Li}^{4,5}$ \\ Meizuo Zhong ${ }^{6}$ \\ Hui Zhou ${ }^{4,5}$ \\ Baohua $\mathrm{Yu}^{\mathrm{I}-3}$ \\ 'Department of Pathology, Fudan \\ University Shanghai Cancer Center, \\ Shanghai, 200032, People's Republic of \\ China; ${ }^{2}$ Department of Oncology, \\ Shanghai Medical College, Fudan \\ University, Shanghai, 200032, People's \\ Republic of China; ${ }^{3}$ Institute of Pathology, \\ Fudan University, Shanghai, 200032, \\ People's Republic of China; ${ }^{4}$ Department \\ of Lymphoma and Hematology, Hunan \\ Cancer Hospital, Changsha, People's \\ Republic of China; ${ }^{5}$ Department of \\ Lymphoma and Hematology, The \\ Affiliated Cancer Hospital of Xiangya \\ School of Medicine, Central South \\ University, Changsha, People's Republic \\ of China; ${ }^{6}$ Department of Oncology, \\ Xiangya Hospital, Central South \\ University, Changsha, Hunan, People's \\ Republic of China
}

Correspondence: Baohua Yu Department of Pathology, Fudan University Shanghai Cancer Center, Shanghai, People's Republic of China Email yubh2014@I63.com

\section{Hui Zhou}

Department of Lymphoma and Hematology, Hunan Cancer Hospital, Changsha, People's Republic of China Email zhouhui9403@I26.com
Objective: Advanced stage extranodal natural killer/T-cell lymphoma (ENKTL) is a distinct type of non-Hodgkin lymphoma and the prognosis of ENKTL is poor with current treatment. This study aimed to investigate the clinical features, treatment strategy and survival outcome in patients with advanced stage ENKTL.

Patients and Methods: A total of 107 patients with newly diagnosed advanced stage ENKTL between January 2010 and December 2014 were reviewed from three cancer centers. Survival probability was calculated using Kaplan-Meier and the survival curves were compared by Log rank test. Cox regression analyses was performed to investigate the prognostic factors in ENKTL.

Results: The median patient age in our cohort was 42.0 years, with a male to female ratio of around 2.3:1. Over half of the patients had B symptoms $(n=61)$, high IPI scores $(\geq 2, n=60)$ and high Prognostic Index of Natural Killer Lymphoma (PINK) scores $(\geq 3, n=69)$. Elevated LDH level was present in around half of the patients (44/91). Most patients $(n=88)$ in our cohort originated in upper aerodigestive tract and the remaining 19 cases presented with non-upper aerodigestive tract involvement at first diagnosis. Chemotherapy regimens used in our study mainly include CHOP (cyclophosphamide, doxorubicin, vincristine, and prednisone) $(\mathrm{n}=26)$, L-asparaginase (L-asp) containing chemotherapy (GELOXD (gemcitabine, 1-asparaginase, oxaliplatin and dexamethasone) and SMILE (L-asparaginase, methotrexate, ifosfamide, etoposide, and dexamethasone)) $(\mathrm{n}=66)$. No significant difference between the baseline clinical characteristics was found between the L-asp and CHOP group. The CR rate after treatment was $39.3 \%(42 / 107)$ for the whole cohort. The 3-year progression-free survival (PFS) and 3-year overall survival (OS) rate was $41.0 \%$ and $41.5 \%$, respectively. The 3 -year PFS $(49.2 \%$ vs $26.5 \%, \mathrm{P}=0.048)$ and 3 -year OS (49.4\% vs $26.0 \%, \mathrm{P}=0.030)$ was significantly higher in the L-asp group than the CHOP group. Patient CR status and PINK score were proved to be significant independent factors affecting OS and PFS by multivariate analysis. The grade $3 / 4$ hematologic toxicity $(\mathrm{P}=0.0003)$ and nonhematologic toxicity $(\mathrm{P}=0.0002)$ occurred more frequently in the SMILE group than the GELOXD group.

Conclusion: Our results demonstrated that L-asp containing chemotherapy could provide favorable survival outcomes in patients with advanced stage ENKTL.

Keywords: extranodal natural killer NK/T-cell lymphoma, chemotherapy, toxicity

\section{Introduction}

Extranodal natural killer/T-cell lymphoma (ENKTL) represents a distinct subtype of non-Hodgkin lymphoma with EBV infection and aggressive clinical course. ENKTL occurs predominantly in the upper aerodigestive tract, with a more 
frequent incidence in Asia and South America than other areas and countries. ${ }^{1}$ Around $20-30 \%$ of the newly diagnosed ENKTL patients were in the advanced stage, with a median survival time of $12-18$ months. ${ }^{2}$ The prognosis of advanced stage ENKTL was poor and no standard therapeutic regimen has been set up for this aggressive lymphoma.

CHOP (cyclophosphamide, doxorubicin, vincristine, and prednisone) is a commonly used regimen in lymphoma, but yields poor results in ENKTL patients due to the overexpression of P-glycoprotein in ENKTL. ${ }^{3-5}$ Recently, L-asparaginase (L-asp)-based chemotherapy such as SMILE (L-asparaginase, methotrexate, ifosfamide, etoposide and dexamethasone) has achieved promising outcomes in advanced stage nasal type ENKTL. ${ }^{6-9}$ However, the above results were based on a relatively small sample size. Here, we summarized the clinicopathologic features, survival outcomes and prognostic factors in advanced stage NK/T-cell lymphoma.

\section{Patients and Methods}

\section{Patient Selection}

Patients diagnosed with advanced stage ENKTL (Ann Arbor stage III/IV disease) at Fudan University Shanghai Cancer Center, Xiangya Hospital and Hunan Cancer Hospital between 2009 and 2014 were systematically reviewed. The inclusion criteria in this study were as follows: (1) pathologic diagnosis of ENKTL based on the WHO classification of lymph and haematogenous tissue tumor; (2) previously untreated, Ann Arbor stage III and IV disease at first diagnosis; (3) a complete set of clinical information, laboratory, treatment and follow-up data; (4) no previous or concomitant malignancies; This study was in accordance with the Declaration of Helsinki and approved by the Ethics Committees of the Fudan University Shanghai Cancer Center, Xiangya Hospital and Hunan Cancer Hospital.

Detailed patient clinic data was collected, including sex, age, systematic B symptoms, a complete medical history and physical examination, serum biochemistries with lactate dehydrogenase (LDH) level, computed tomography (CT) and/or magnetic resonance imaging of the head and neck, computed tomography of the chest, abdomen and pelvis, or positron emission tomographycomputed tomography (PET/CT); bone marrow examination.
New prognostic model Prognostic Index of Natural Killer Lymphoma (PINK) was evaluated in our study. PINK represents a new prognostic index based on four prognostic factors, ${ }^{10}$ a patient aged $>60$ years, tumor stage III-IV disease, disease with distant lymph node involvement, and non-nasal type disease.

\section{Treatment Protocol}

GELOXD was a three-week cycle regimen, including gemcitabine $\left(800 \mathrm{mg} / \mathrm{m}^{2}, \mathrm{~d} 1,5\right)$, l-asparaginase $\left(5000 \mathrm{U} / \mathrm{m}^{2}, \mathrm{~d} 1\right.$ 7), oxaliplatin $\left(85 \mathrm{mg} / \mathrm{m}^{2}, \mathrm{~d} 1\right)$ and dexamethasone $(10 \mathrm{mg}$, on d1-7). VIPD was a 21-day cycle that included etoposide $\left(75 \mathrm{mg} / \mathrm{m}^{2}\right)$, ifosfamide $\left(75 \mathrm{mg} / \mathrm{m}^{2}\right)$, cisplatin $\left(20 \mathrm{mg} / \mathrm{m}^{2}\right)$ and dexamethasone (40 mg) on days $1-4$. Modified SMILE regimen included L-asparaginase $\left(6000 \mathrm{U} / \mathrm{m}^{2}\right)$ on days $1-7$, methotrexate $\left(60 \mathrm{mg} / \mathrm{m}^{2}\right)$ on day 1 , ifosfamide $\left(1.5 \mathrm{~g} / \mathrm{m}^{2}\right)$ on days $2-4$, etoposide $\left(100 \mathrm{U} / \mathrm{m}^{2}\right)$ on days $2-4$, and dexamethasone $(15 \mathrm{mg})$ on days $1-7$, with a cycle of 21 days. All the patients received 4-6 cycles of chemotherapy.

\section{Evaluation Standards}

Based on the revised response criteria for lymphoma, ${ }^{11}$ the response of the patients in our cohort was categorized as complete response (CR), partial response (PR), stable disease (SD) or progressive disease (PD). PET/CT, MRI and/ or CT were performed before treatment, after every two cycles of chemotherapy, after treatment, and to monitor relapses.

\section{Evaluation of Adverse Effects}

The adverse effects of chemotherapy were evaluated according to the National Cancer Institute Common Toxicity Criteria (version 3.0). Based on this criteria, the treatment-related toxicity was classified into grade 1 to 5 .

\section{Data and Survival Analysis}

Progression-free survival (PFS) was measured from the time of diagnosis to the time of disease progression, relapse, or death by any cause. Overall survival (OS) was calculated from the date of diagnosis to the date of the last follow-up or death. The survival data was analyzed by Kaplan-Meier method and Log rank test was used to compare the survival curves. Significant factors in univariate analysis $(\mathrm{P}<0.05)$ were further evaluated by multiple factors analysis with Cox regression model. The difference of categorical variables between two groups was compared by Pearson Chisquare analysis or Fisher exact test. $\mathrm{P}<0.05$ were considered statistically significant and all the statistical analyses were 
performed using SPSS 18.0 software SPSS 19.0 software (SPSS Inc, Chicago, IL, USA) and GraphPad Prism 5 software.

\section{Results}

\section{Patient Characteristics}

A total of 107 patients with newly diagnosed advanced stage ENKTL were recruited from three cancer centers. The detailed clinical features and immunohistochemical results are summarized in Table 1. The median patient age was 42.0 years (range, 10-76 years) and most patients in our cohort were young and middle-aged adults, with a male to female ratio of around 2.3:1. A vast majority of patients in our cohort had a good ECOG PS $(\leq 1, \mathrm{n}=105)$ and over half of the patients had B symptoms ( $n=61)$, high IPI scores $(\geq 2$, $n=60)$ and high PINK scores $(\geq 3, n=69)$. Elevated LDH level was present in around half of patients (44/91) at first diagnosis. Most patients $(n=88)$ in our cohort originated in upper aerodigestive tract, including the nasal cavity $(n=70)$, nasopharynx $(\mathrm{n}=14)$, and oropharynx $(\mathrm{n}=4)$. Non-upper aerodigestive tract (NUAT) involvement was present in 19 patients, including skin $(\mathrm{n}=9)$, intestinal tract $(\mathrm{n}=6)$,

Table I Patient Characteristics

\begin{tabular}{|c|c|c|c|c|c|c|}
\hline \multirow[t]{2}{*}{ Characteristics } & \multirow{2}{*}{$\begin{array}{c}\text { Patient Number } \\
\text { (\%) }\end{array}$} & \multirow{2}{*}{$\begin{array}{l}\text { CHOP Group } \\
(n=28)\end{array}$} & \multicolumn{3}{|c|}{ L-asp Group $(n=66)$} & \multirow{2}{*}{$\begin{array}{c}\text { P value (L-asp vs CHOP } \\
\text { Group) }\end{array}$} \\
\hline & & & Total & GELOXD $(n=44)$ & SMILE $(n=22)$ & \\
\hline Gender & & & & & & 0.4237 \\
\hline Male & $75(70.1)$ & 21 & 44 & 28 & 16 & \\
\hline Female & $32(29.9)$ & 7 & 22 & 16 & 6 & \\
\hline Age (years) & & & & & & 0.9702 \\
\hline$>60$ & $20(18.7)$ & 5 & 12 & 7 & 05 & \\
\hline$\leq 60$ & $87(81.3)$ & 23 & 54 & 37 & 017 & \\
\hline Median (years) & 42.0 & 37.5 & 41.5 & & & \\
\hline ECOG PS & & & & & & 0.5275 \\
\hline $0-1$ & $105(98.1)$ & 27 & 65 & 43 & 022 & \\
\hline $2-4$ & $2(1.9)$ & 1 & 1 & 1 & 0 & \\
\hline B symptom & & & & & & 0.2049 \\
\hline Yes & $61(57.0)$ & 13 & 40 & 25 & 015 & \\
\hline No & $46(43.0)$ & 15 & 26 & 19 & 07 & \\
\hline IPI score & 91 & 23 & 60 & 41 & 019 & 0.1642 \\
\hline$>1$ & $31(34.1)$ & 16 & 50 & 36 & 014 & \\
\hline$\leq 1$ & $60(65.9)$ & 7 & 10 & 5 & 05 & \\
\hline LDH level & 91 & 23 & 60 & 40 & 020 & 0.3745 \\
\hline Elevated & $44(48.4)$ & 9 & 30 & 17 & 013 & \\
\hline Normal & $47(51.6)$ & 14 & 30 & 23 & 07 & \\
\hline Primary sites & & & & & & 0.5596 \\
\hline UAT & $88(82.2)$ & 23 & 52 & 36 & 017 & \\
\hline NAT & $19(17.8)$ & 4 & 13 & 8 & 05 & \\
\hline Skin & 9 & 2 & 5 & 3 & 02 & \\
\hline Intestinal tract & $6(1.4)$ & 1 & 5 & 3 & 02 & \\
\hline Breast & 1 & 0 & 1 & 1 & 00 & \\
\hline Distant lymph nodes & 3 & 1 & 2 & 1 & 01 & \\
\hline PINK & 107 & 28 & 66 & & & 0.2282 \\
\hline $1-2$ & $38(35.5)$ & 7 & 25 & 13 & 012 & \\
\hline$\geq 3$ & $69(64.5)$ & 21 & 41 & 31 & 010 & \\
\hline \multicolumn{7}{|l|}{ Immunophenotype } \\
\hline $\mathrm{Ki}-67 \geq 60 \%$ & $62(57.9)$ & 18 & 32 & 22 & 010 & 0.1603 \\
\hline EBER + & I0I (94.4) & 25 & 62 & 41 & 021 & 0.4319 \\
\hline
\end{tabular}

Abbreviations: ECOG PS, Eastern Cooperative Oncology Group performance status; IPI, International Prognostic Index; LDH, lactate dehydrogenase; PINK, Prognostic Index of Natural Killer Lymphoma; EBER, EBV encoded RNA. 
distant lymph nodes $(n=3)$, and breast $(n=1)$. The median $\mathrm{Ki}-67$ value was $60 \%$ in the current study, with a wide range between $10 \%$ and $100 \%$. Ten patients were positive for HBsAg and antiviral medication was administered before chemotherapy and radiotherapy in these patients. There is no obvious difference between the baseline clinical characteristics for the CHOP group and the L-asp group.

\section{Evaluation of Efficacy and Survival Outcomes}

Of the 107 patients, 101 received chemotherapy as first line treatment, and two patients did not receive treatment due to disease progression or other personal reasons. One patient only received radiotherapy alone as a palliative therapy. The chemotherapy regimens used in our cohort were as follow: GELOXD for 44 cases, SMILE for 22 cases, VIPD for 7 cases, CHOP for 28 cases. The remaining three patients received other regimens, including EPOCH and ATT. The CR rate after treatment was $39.3 \%(42 / 107)$ for the whole cohort. Of the 101 stage III/IV patients treated with chemotherapy, CR rate and ORR were 39.6\% (40/101) and 63.4\% (64/101), respectively. Compared with the CHOP group, the $\mathrm{CR}$ rate (47.0\% vs $21.4 \%, p=0.0204)$ and ORR rate $(68.2 \%$ vs $42.9 \%, p=0.0215)$ were significantly higher in the L-asp group (GELOXD and SMILE) (Table 2).

The median follow-up time was 30.5 months, with a range of 1-86 months. The 3-year PFS and 3-year OS rate was $41.0 \%$ (95\% CI, 5.4-34.6\%) and $41.5 \%$ (95\% CI, 12.3-35.7\%) respectively for the whole cohort (Figure 1A and $\mathrm{B})$. The 3 -year PFS (49.2\% vs $26.5 \%, \mathrm{P}=0.048)$ and 3 -year OS (49.4\% vs $26.0 \%, \mathrm{P}=0.030)$ was significantly higher in the L-asp group than CHOP group (Figure $1 \mathrm{C}$ and D). However, no survival difference was found between GELOXD and SMILE group (3-year PFS 47.7\% vs 51.4\%, $\mathrm{p}=0.9455$; 3-year OS $45.9 \%$ vs $55.4 \%, \mathrm{p}=0.5435$ ) (Figure $1 \mathrm{E}$ and F). Notably, patients with the primary involved site of upper aerodigestive tract (UAT) obtained higher 3-year PFS $(44.6 \%$ vs. $18.3 \%, \mathrm{P}=0.002)$ and $\mathrm{OS}(45.1 \%$ vs $19.8 \%$,
$\mathrm{P}=0.012)$ than those with non-upper aerodigestive tract (NUAT) (Figure 2A and B).

IPI and PINK were a significant prognostic factor in our study (Figure 2C-F). Patients in the high-risk groups (PINK $\geq 3$ ) had worse PFS (3-year PFS 50.7\% vs $11.9 \%, \mathrm{P}=0.001$ ) and OS (3-year OS $49.1 \%$ vs $15.7 \%, \mathrm{P}=0.011$ ) rates than the low-risk $(\mathrm{PINK}<3)$ group (Figure $2 \mathrm{E}$ and $2 \mathrm{~F}$ ).

\section{Prognostic Factors for PFS and OS}

In our study, univariate analysis showed that some significant factors could affect PFS, including age $(\mathrm{P}=0.011)$, primary tumor site $(\mathrm{P}=0.002)$, IPI score $(\mathrm{P}=0.029)$, PINK score $(P=0.001)$ and $C R$ status $(P<0.001)$. Patient CR status $(\mathrm{P}=0.002)$ and PINK score $(\mathrm{P}=$ 0.014) were important independent factors affecting PFS by multivariate analysis (Table 3 ).

Significant factors affecting OS by univariate analysis included patient age $(\mathrm{P}=0.048)$, primary tumor site $(\mathrm{P}=$ 0.012), IPI score $(\mathrm{P}=0.039)$, PINK score $(\mathrm{P}=0.011)$, L-asp containing chemotherapy $(\mathrm{P}=0.042)$ and patient $\mathrm{CR}$ status $(\mathrm{P}<0.001)$. Multivariate analysis demonstrated that PINK score $(\mathrm{P}=0.020)$ and $\mathrm{CR}$ status $(\mathrm{P}=0.003)$ were significant independent factors for the whole cohort (Table 3).

\section{Adverse Effects}

Table 4 summarizes the treatment-related adverse effects of the GELOXD and SMILE regimen. The most common adverse effect of these two regimens was hematological toxicity and the SMILE regimen had significantly more severe toxicity. A relatively high rate of grade 3/4 hematologic toxicity was observed in the SMILE group, including neutropenia $(\mathrm{n}=12$, $54.5 \%)$, thrombocytopenia $(\mathrm{n}=9,40.9 \%)$, anemia $(\mathrm{n}=8$, $36.4 \%)$ and febrile neutropenia $(n=2,9.1 \%)$, followed by nonhematological toxicities including nausea $(\mathrm{n}=8,36.4 \%)$, transaminase elevation $(\mathrm{n}=7,31.8 \%)$, hyperbilirubinemia $(\mathrm{n}$ $=3,13.6 \%)$, vomiting $(\mathrm{n}=3,13.6 \%)$, diarrhea $(\mathrm{n}=2,9.1 \%)$, fatigue $(n=3,13.6 \%)$ and peripheral neuropathy $(n=2,9.1 \%)$. The toxicities of GELOXD were mild and the most frequent

Table 2 The Response Rates for Different Treatment Regimens

\begin{tabular}{|l|c|c|c|c|c|}
\hline & CHOP (n=28) & L-asp Containing Regimen (n=66) & VIPD (n=7) & P value (L-asp vs CHOP) & P value (VIPD vs CHOP) \\
\hline CR & 6 & 31 & 3 & 0.0204 & 0.2460 \\
PR & 6 & 14 & 2 & & \\
SD & 4 & 9 & 1 & & \\
PD & 12 & 12 & $71.4 \%$ & 0.0215 & 0.2285 \\
ORR & $42.9 \%$ & $68.2 \%$ & & & \\
\hline
\end{tabular}


A

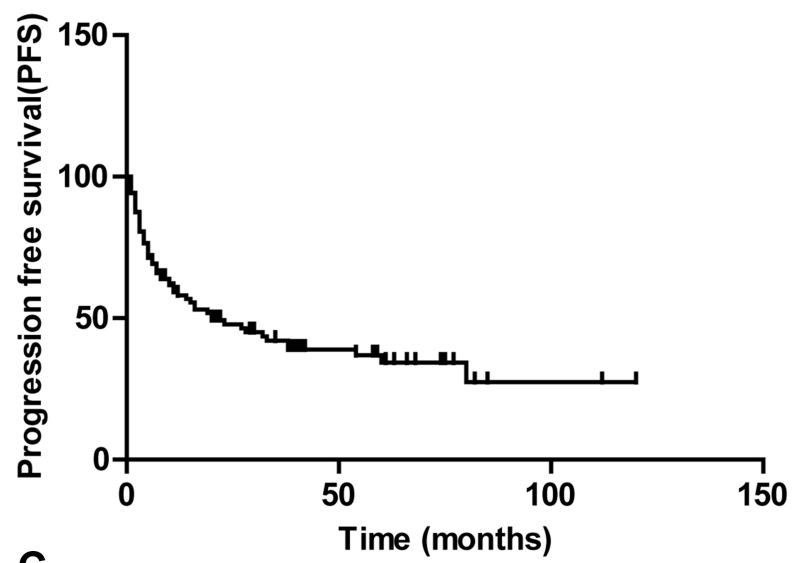

C

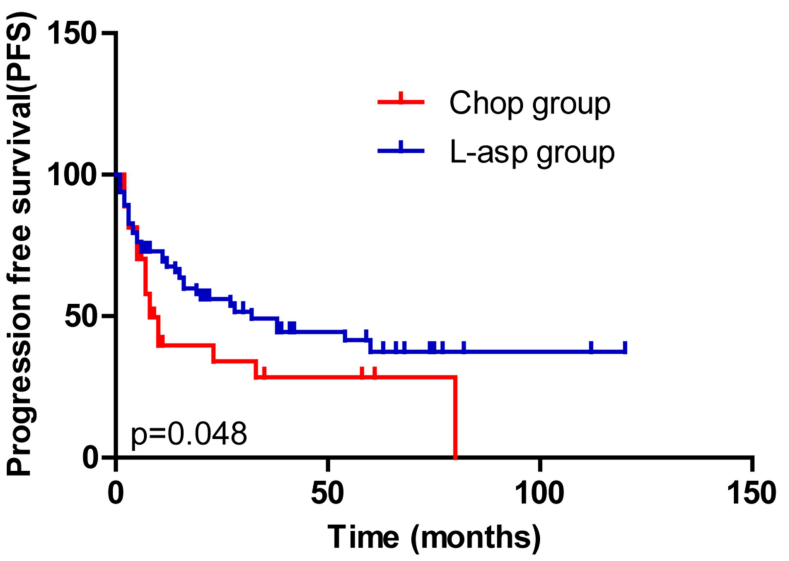

$\mathbf{E}$

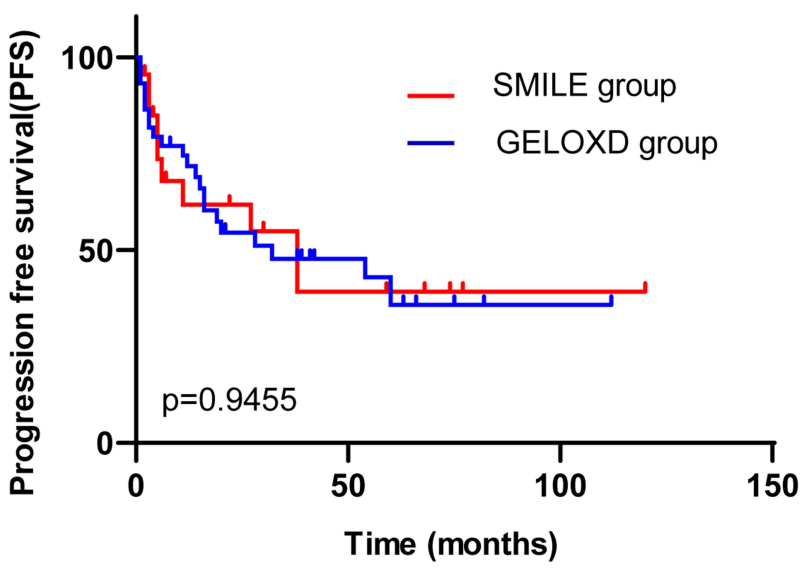

B
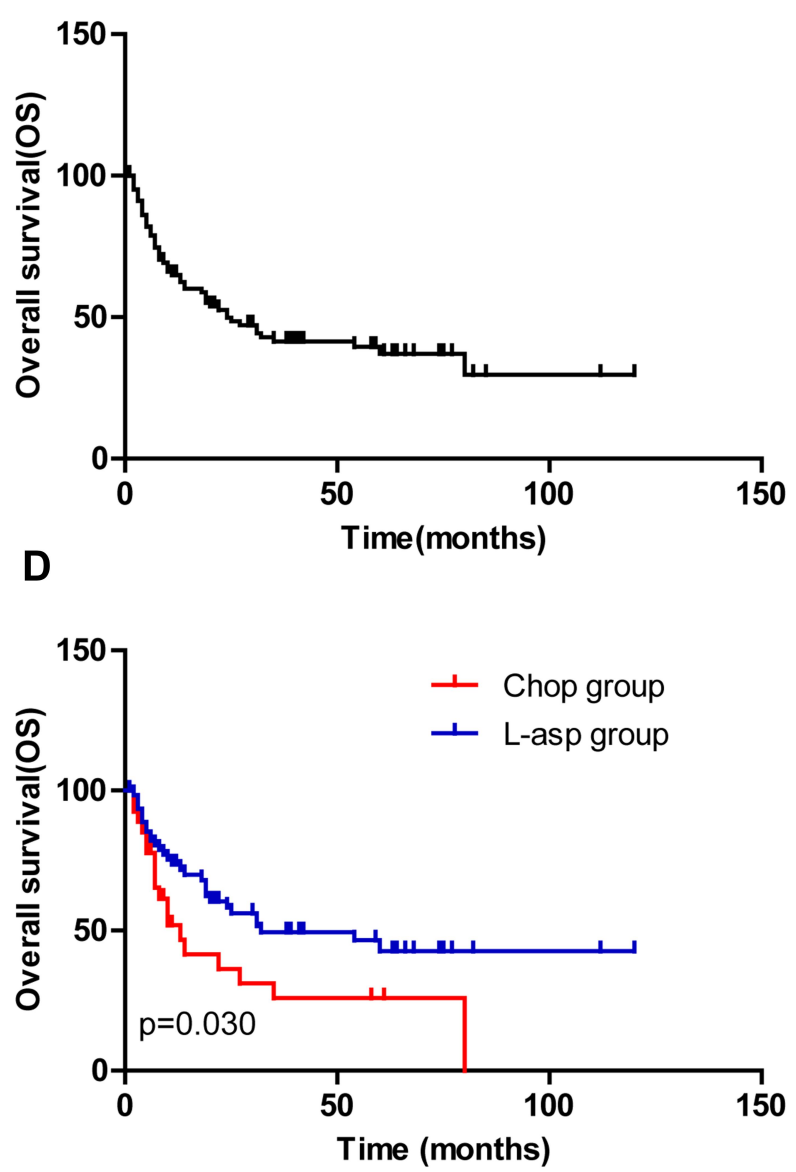

$\mathbf{F}$

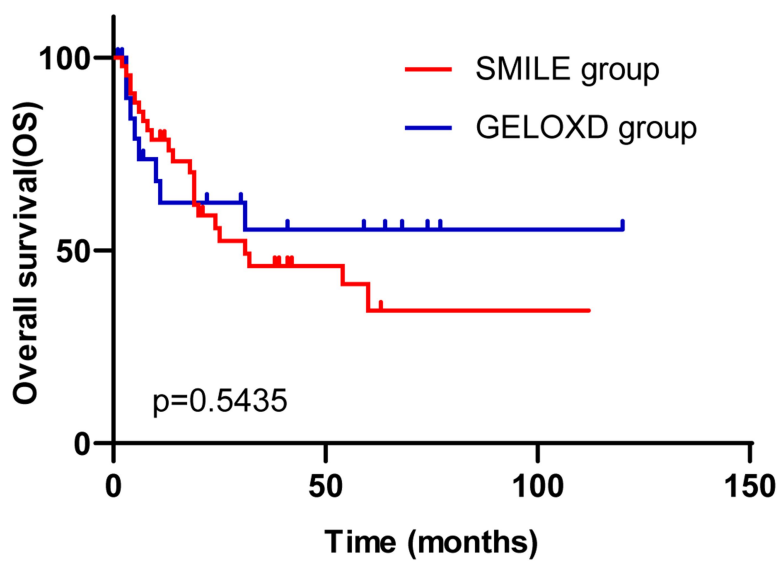

Figure I Kaplan-Meier survival curve of advanced stage ENKTL patients.

Notes: (A and B) The progression-free survival (PFS) (A) and overall survival (OS) (B) of all patients. (C) The progression-free survival in the CHOP and L-asp groups. (D) The overall survival in the CHOP and L-asp groups. (E) The progression-free survival in the SMILE and GELOXD groups. (F) The overall survival in the SMILE and GELOXD groups.

grade $3 / 4$ toxicities were neutropenia $(n=6,13.6 \%$ ), nausea $(\mathrm{n}=5,11.4 \%)$ and anemia $(\mathrm{n}=4,9.1 \%)$.

\section{Discussion}

Extranodal NK/T-cell lymphoma represents a distinct entity of non-Hodgkin lymphoma characterized by predilection of midline facial tissues involvement, high rate of EBV infection and aggressive clinic course. ${ }^{8}$ The prognosis of advanced stage ENKTL remains poor with conventional chemotherapy and application of L-asp shedding light on the treatment of ENKTL. ${ }^{6,8}$ In this study, we summarized our experience of advanced stage ENKTL. 

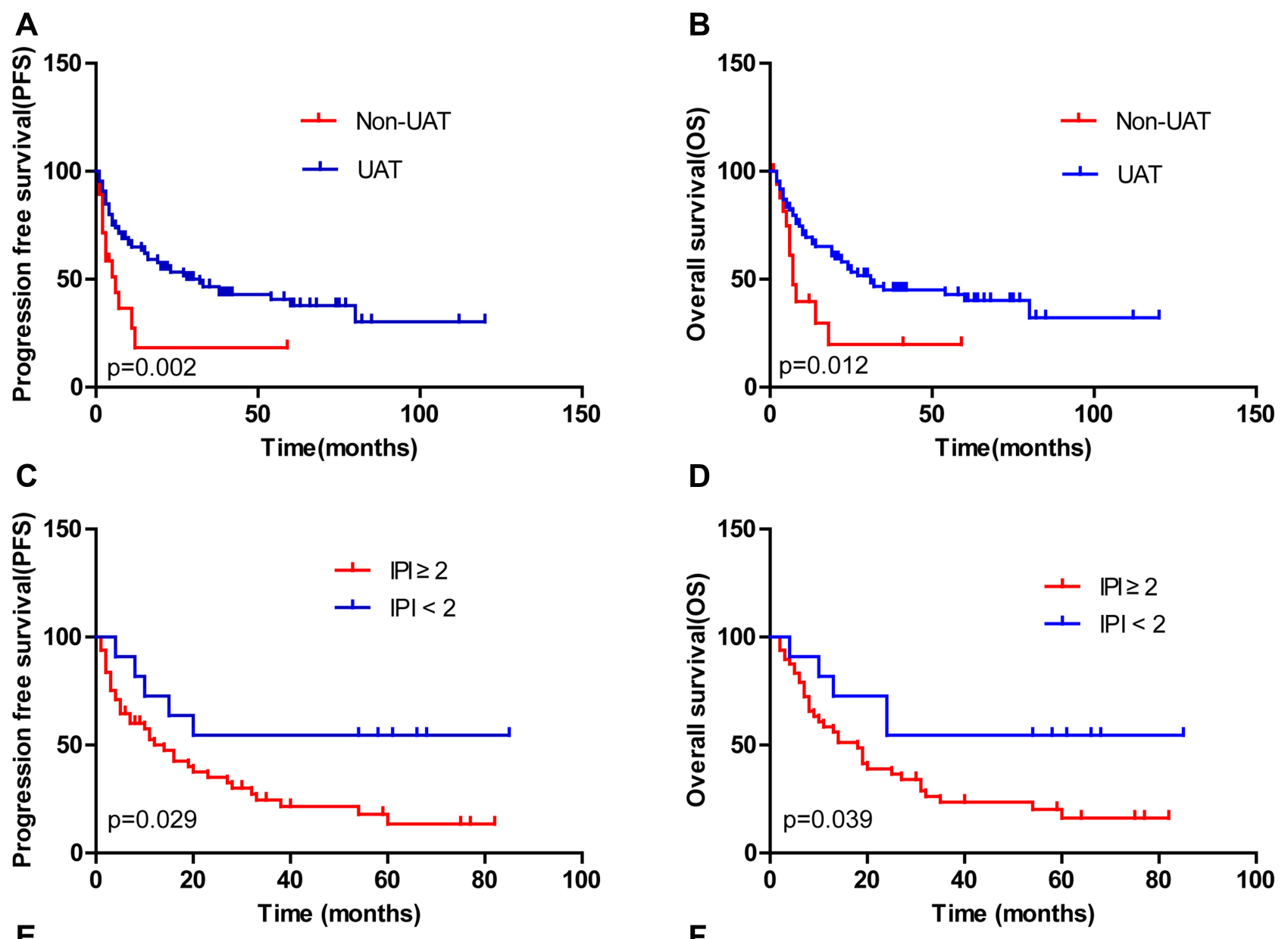

D
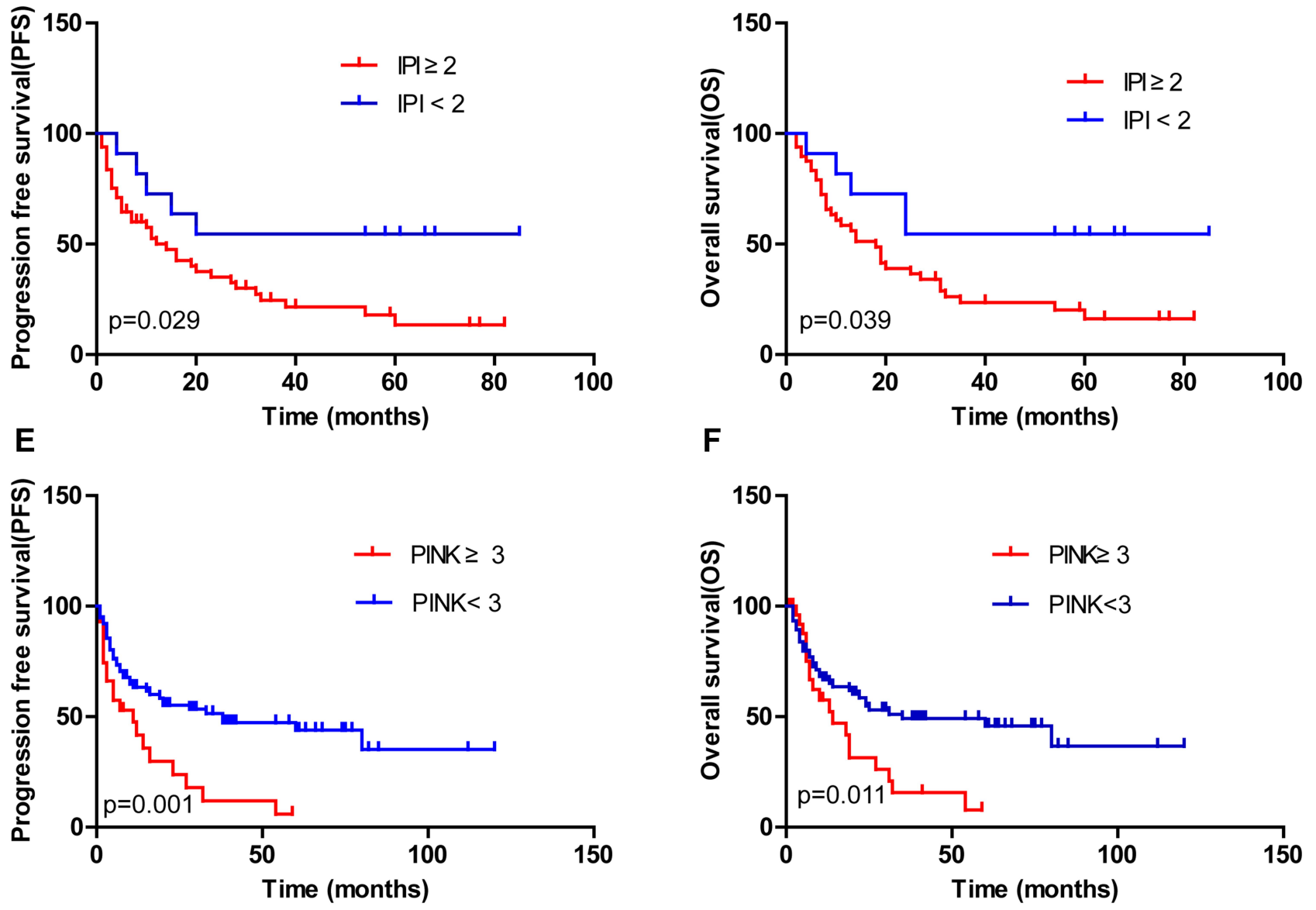

Figure 2 Significant impact of primary involved site, IPI score and PINK score on the survival outcome.

Notes: (A) The progression-free survival in the UAT and NUAT groups. (B) The overall survival in the UAT and NUAT groups. (C) The progression-free survival in the lowIPI and high-IPI groups. (D) The overall survival in the low-IPI and high-IPI groups. (E) The progression-free survival in the low-PINK and high-PINK groups. (F) The overall survival in the low-PINK and high-PINK groups.

Anthracycline-based chemotherapy was a common regimen for non-Hodgkin lymphoma and once used in advanced stage ENKTL. However, previous studies demonstrated the CHOP regimen yields poor results in advanced stage ENKTL, with a low CR rate and short survival time. ${ }^{4,5} \mathrm{Kim}$ et $\mathrm{al}^{4}$ reported the results of 59 ENKTL patients treated with anthracycline-based chemotherapy. In this study, 41 stage I/II and 18 stage III/IV ENKTL patients received anthracycline-based chemotherapy as first-line treatment, after a median follow up time of 
Table 3 Univariate and Multivariate Analysis of Prognostic Factors for Survivals (by Cox Regression)

\begin{tabular}{|c|c|c|c|c|c|c|c|c|}
\hline \multirow[t]{3}{*}{ Clinical Factor } & \multicolumn{4}{|c|}{ Progression-Free Survival } & \multicolumn{4}{|c|}{ Overall Survival } \\
\hline & \multicolumn{2}{|c|}{ Univariate } & \multicolumn{2}{|c|}{ Multivariate } & \multicolumn{2}{|c|}{ Univariate } & \multicolumn{2}{|c|}{ Multivariate } \\
\hline & $P$ & HR (95\% Cl) & $P$ & HR (95\% Cl) & $P$ & HR (95\% Cl) & $P$ & HR (95\% Cl) \\
\hline Aged $\geq 60 y$ & 0.011 & $0470(0.255-0.865)$ & & & 0.019 & $0.491(0.264-0.909)$ & & \\
\hline Gender & 0.817 & $0.921(0.458-1.852)$ & & & 0.820 & $0.919(0.444-1.903)$ & & \\
\hline B symptom & 0.928 & $1.029(0.550-1.924)$ & & & 0.909 & $0.963(0.502-1.847)$ & & \\
\hline LDH & 0.532 & $1.243(0.628-2.462)$ & & & 0.649 & $1.173(0.648-2.156)$ & & \\
\hline $\mathrm{Ki} 67 \geq 60 \%$ & 0.238 & $0.605(0.262-1.395)$ & & & 0.553 & $0.763(0.313-1.862)$ & & \\
\hline Primary tumor site (UAT vs NUAT) & 0.002 & $0.377(0.195-0.728)$ & & & 0.012 & $0.435(0.221-0.855)$ & & \\
\hline Stage (III vs IV) & 0.147 & $1.837(0.807-4.180)$ & & & 0.238 & $1.648(0.719-3.778)$ & & \\
\hline IPI score $\geq 2$ & 0.029 & $0.370(0.144-0.95 I)$ & & & 0.039 & $0.389(0.151-0.999)$ & & \\
\hline PINK score $\geq 3$ & 0.001 & $0.417(0.239-0.726)$ & 0.014 & $0.335(0.141-0.798)$ & 0.011 & $0.491(0.279-0.866)$ & 0.048 & $0.415(0.174-0.991)$ \\
\hline$\beta 2$-microglobulin elevated $>2.5 \mathrm{mg} / \mathrm{L}$ & 0.974 & $0.986(0.416-2.333)$ & & & 0.770 & $0.874(0.355-2.154)$ & & \\
\hline Hemoglobin < IIOg/L & 0.568 & $0.828(0.420-1.632)$ & & & 0.289 & $0.677(0.329-1.391)$ & & \\
\hline Leukopenia $<4 \times 10^{9} / \mathrm{L}$ & 0.415 & $1.359(0.650-2.842)$ & & & 0.732 & $1.140(0.538-2.419)$ & & \\
\hline Platelet $<150 \times 10^{9} / \mathrm{L}$ & 0.649 & $0.853(0.431-1.690)$ & & & 0.367 & $0.723(0.358-|.46|)$ & & \\
\hline L-asp containing regimen & 0.106 & $1.548(0.899-2.667)$ & & & 0.042 & $1.776(1.022-3.089)$ & & \\
\hline $\mathrm{CR}$ after treatment & 0.000 & $5.409(2.893-10.113)$ & 0.002 & $4.577(1.724 \mid 2.150)$ & 0.000 & $5.150(2.717-9.759)$ & 0.045 & $2.507(1.021-6.153)$ \\
\hline
\end{tabular}

27.2 months, the 2-year disease-free survival and 2-year overall survival were $22.9 \%$ and $44.2 \%$, respectively. Similarly, the efficacy of the CHOP regimen in our study was disappointing, with a $\mathrm{CR}$ rate of $23.1 \%$ and 3 -year OS of $26.0 \%$. The overexpression of P-gp overexpressed by lymphoma cells may contribute to the drug resistance. ${ }^{12,13}$

As tumor cells lack L-asparagine synthetase, L-asp could produce anticancer effects by hydrolyzing serum asparagine, which was necessary for the survival of lymphoma cells. As such, L-Asp was not affected by multidrug resistance and proved to be effective in advanced

Table 4 Grade 3/4 Toxicity That Occurred in the Two Groups

\begin{tabular}{|l|c|c|c|}
\hline Toxicity & $\begin{array}{c}\text { GELOXD } \\
(\mathbf{n = 4 4 )}\end{array}$ & $\begin{array}{c}\text { SMILE } \\
(\mathbf{n}=\mathbf{2 2})\end{array}$ & P value \\
\hline Hematologic & 7 & 13 & 0.0003 \\
Neutropenia & $6(13.6 \%)$ & $12(54.5 \%)$ & \\
Anemia & $4(9.1 \%)$ & $8(36.4 \%)$ & \\
Thrombocytopenia & $2(4.5 \%)$ & $9(40.9 \%)$ & \\
Febrile neutropenia & $1(2.3 \%)$ & $2(9.1 \%)$ & \\
\hline Non-hematologic & 8 & 14 & \multirow{2}{*}{0.0002} \\
Nausea & $5(11.4 \%)$ & $8(36.4 \%)$ & \\
Vomiting & $2(4.5 \%)$ & $3(13.6 \%)$ & \\
Diarrhea & 0 & $2(9.1 \%)$ & \\
Transaminase elevation & $2(4.5 \%)$ & $7(31.8 \%)$ & \\
ALT elevation & $2(4.5 \%)$ & $3(13.6 \%)$ & \\
AST elevation & $1(2.3 \%)$ & $5(22.7 \%)$ & \\
Hyperbilirubinemia & $1(2.3 \%)$ & $3(13.6 \%)$ & \\
Peripheral neuropathy & $1(2.3 \%)$ & $2(9.1 \%)$ & \\
Fatigue & $1(2.3 \%)$ & $3(13.6 \%)$ & \\
\hline
\end{tabular}

stage ENKTL. ${ }^{6,714-16}$ Previous studies reported that some L-Asp based chemotherapy such as GELOX, SMILE and DDGP obtained good efficacy in advanced stage ENKTL. ${ }^{6-8}$ A multicenter prospective study including 42 advanced ENKTL patients demonstrated that the DDGP group had a better 2 year OS (74\% versus $45 \%$, $\mathrm{P}=0.027)$ than the SMILE group, indicating that L-asp containing chemotherapy was effective in advanced ENKTL. ${ }^{7}$ Consistent with the above study, our results showed that the L-asp based regimen acquired a good therapeutic effect in patients with advanced ENKTL.

Some risk factors, including clinical stage, B symptoms, LDH, IPI score, PINK score and patient CR status, were proved to have been important prognostic factors in the last few years. ${ }^{16,17}$ Our study demonstrated that primary involved site, patient age, IPI score, PINK score, non-anthracycline containing chemotherapy and patient CR status were significant factors affecting survival outcome by univariate analysis. However, only IPI score and CR status remained significant independent factors affecting PFS or OS in multivariate analysis.

The primary tumor site was an important factor affecting survival outcome. Several studies have demonstrated that stage III/IV ENKTL patients with primary site of upper aerodigestive tract have a more favorable clinical outcome than those originating in non-upper aerodigestive tract. ${ }^{18}$ Similar to these researches, our result showed that the response rate and 3-year survival rate was significantly higher in patients with UAT advanced stage ENKTL, 
indicating that this unique group of patients may be different in the pathogenesis and need to be explored as a single entity.

PINK score was a new prognostic model for ENKTL treated with non-anthracycline-based therapies. ${ }^{10}$ In our study, the PINK score was an important factor affecting OS and PFS by univariate analysis, consistent with previous reports. $^{16,19}$

Treatment-related toxicity of GELOXD and SMILE was evaluated in the present study. Treatment-related toxicity was mild in GELOXD, which was similar to a previous study, which demonstrated that $30 \%$ of cases had grade $3 / 4$ neutropenia and $8.5 \%$ had grade $3 / 4$ hepatotoxicity. ${ }^{20}$ Compared with the GELOXD group, the SMILE group had significantly more severe hematological toxicities and liver dysfunctions in our study. However, the toxicity of SMILE was milder than a previous study, in which grade $3 / 4$ neutropenia was observed in $100 \%$ of patients. ${ }^{6}$ The possible reason may be the relatively low dose of etoposide and cisplatin used in our study.

\section{Conclusion}

In conclusion, our study showed that the L-asp containing regimen could produce a higher $\mathrm{CR}$ rate and a longer survival time than the CHOP regimen in advanced stage ENKTL. The favorable outcome in our study demonstrated that GELOXD and SMILE are effective in patients with newly diagnosed advanced stage ENKTL. However, the present study also showed some limitations, such as heterogeneity of the treatment protocols and retrospective design of the study. Prospective and multicenter studies are needed to evaluate the efficacy and tolerance of the L-asp containing regimen and VIPD regimen in ENKTL patients in the future.

\section{Funding}

This work was supported by the Youth Project of the National Natural Science Foundation of China (No.81700195), the "Scientific Research Climbing Plan" of Hunan Cancer Hospital [no. ZX2020003], the Research Program of Hunan Provincial Health and Family Planning Commission [no: B20180496], the Changsha Science and Technology Plan [no: kq1706041].

\section{Disclosure}

The authors report no conflicts of interest in this work.

\section{References}

1. Aozasa K, Takakuwa T, Hongyo T, Yang WI. Nasal NK/T-cell lymphoma: epidemiology and pathogenesis. Int J Hematol. 2008;87 (2):110-117. doi:10.1007/s12185-008-0021-7

2. Lee J, Suh C, Park YH, et al. Extranodal natural killer T-cell lymphoma, nasal-type: a prognostic model from a retrospective multicenter study. J Clin Oncol. 2006;24(4):612-618. doi:10.1200/JCO.2005.04.1384

3. Wang L, Xia ZJ, Huang HQ, Lu Y, Zhang YJ. Cyclophosphamide, doxorubicin, vincristine, and prednisone (CHOP) in the treatment of stage IE/IIE extranodal natural killer/T cell lymphoma, nasal type: 13-year follow-up in 135 patients. Int J Hematol. 2012;96 (5):617-623. doi:10.1007/s12185-012-1174-y

4. Kim BS, Kim TY, Kim CW, et al. Therapeutic outcome of extranodal NK/T-cell lymphoma initially treated with chemotherapy-result of chemotherapy in NK/T-cell lymphoma. Acta Oncol. 2003;42 (7):779-783. doi:10.1080/02841860310010682

5. Suzuki R, Suzumiya J, Yamaguchi M, et al. Prognostic factors for mature natural killer (NK) cell neoplasms: aggressive NK cell leukemia and extranodal NK cell lymphoma, nasal type. Ann Oncol. 2010;21(5):1032-1040. doi:10.1093/annonc/mdp418

6. Yamaguchi M, Kwong YL, Kim WS, et al. Phase II study of SMILE chemotherapy for newly diagnosed stage IV, relapsed, or refractory extranodal natural killer (NK)/T-cell lymphoma, nasal type: the NK-cell tumor study group study. J Clin Oncol. 2011;29 (33):4410-4416. doi:10.1200/JCO.2011.35.6287

7. Li X, Cui Y, Sun Z, et al. DDGP versus SMILE in newly diagnosed advanced natural killer/T-cell lymphoma: a randomized controlled, multicenter, open-label study in China. Clin Cancer Res. 2016;22 (21):5223-5228. doi:10.1158/1078-0432.CCR-16-0153

8. Yamaguchi M, Suzuki R, Oguchi M. Advances in the treatment of extranodal NK/T-cell lymphoma, nasal type. Blood. 2018;131 (23):2528-2540. doi:10.1182/blood-2017-12-791418

9. Wang JH, Wang L, Liu CC, et al. Efficacy of combined gemcitabine, oxaliplatin and pegaspargase (P-gemox regimen) in patients with newly diagnosed advanced-stage or relapsed/refractory extranodal NK/T-cell lymphoma. Oncotarget. 2016;7(20):29092-29101. doi:10.18632/oncotarget.8647

10. Kim SJ, Yoon DH, Jaccard A, et al. A prognostic index for natural killer cell lymphoma after non-anthracycline-based treatment: a multicentre, retrospective analysis. Lancet Oncol. 2016;17 (3):389-400. doi:10.1016/S1470-2045(15)00533-1

11. Cheson BD, Pfistner B, Juweid ME, et al. Revised response criteria for malignant lymphoma. J Clin Oncol. 2007;25(5):579-586. doi:10.1200/JCO.2006.09.2403

12. Yamaguchi M, Kita K, Miwa $\mathrm{H}$, et al. Frequent expression of P-glycoprotein/MDR1 by nasal T-cell lymphoma cells. Cancer. 1995;76(11):2351-2356. doi:10.1002/1097-0142(19951201) 76:11<2351::aid-cncr2820761125>3.0.co;2-1

13. Wang B, Li XQ, Ma X, Hong X, Lu H, Guo Y. Immunohistochemical expression and clinical significance of P-glycoprotein in previously untreated extranodal NK/T-cell lymphoma, nasal type. Am J Hematol. 2008;83(10):795-799. doi:10.1002/ajh.21256

14. Lin N, Song Y, Zheng W, et al. A prospective phase II study of L-asparaginase- CHOP plus radiation in newly diagnosed extranodal NK/T-cell lymphoma, nasal type. J Hematol Oncol. 2013;6(1):44. doi:10.1186/1756-8722-6-44

15. Yang L, Liu H, Xu XH, et al. Retrospective study of modified SMILE chemotherapy for advanced-stage, relapsed, or refractory extranodal natural killer (NK)/T cell lymphoma, nasal type. Med Oncol. 2013;30 (4):720. doi:10.1007/s12032-013-0720-7

16. Li JW, Li YJ, Zhong MZ, et al. Efficacy and tolerance of GELOXD/ P-GEMOXD in newly diagnosed nasal-type extranodal NK/T-cell lymphoma: a multicenter retrospective study. Eur J Haematol. 2018;100(3):247-256. doi:10.1111/ejh.13004 
17. Kim TM, Lee SY, Jeon YK, et al. Clinical heterogeneity of extranodal NK/T-cell lymphoma, nasal type: a national survey of the Korean cancer study group. Ann Oncol. 2008;19(8):1477-1484. doi:10.1093/annonc/mdn147

18. Yao YY, Tang Y, Zhuang Y, et al. Retrospective Study of Pegaspargase, Gemicitabine, Oxaliplatin and Dexamethasone (Peg-GemOD) as a first-line therapy for advanced-stage extranodal NK/T cell lymphoma. Indian $J$ Hematol Blood Transfus. 2017;33(1):74-81. doi:10.1007/s12288-016-06 $70-2$
19. Chen SY, Yang Y, Qi SN, et al. Validation of nomogram-revised risk index and comparison with other models for extranodal nasal-type NK/T-cell lymphoma in the modern chemotherapy era: indication for prognostication and clinical decision-making. Leukemia. 2021;35 (1):130-142. doi:10.1038/s41375-020-0791-3

20. Wang JH, Wang H, Wang YJ, et al. Analysis of the efficacy and safety of a combined gemcitabine, oxaliplatin and pegaspargase regimen for NK/T-cell lymphoma. Oncotarget. 2016;7(23):35412-35422. doi:10.18632/oncotarget. 8643

\section{Publish your work in this journal}

Cancer Management and Research is an international, peer-reviewed open access journal focusing on cancer research and the optimal use of preventative and integrated treatment interventions to achieve improved outcomes, enhanced survival and quality of life for the cancer patient.
The manuscript management system is completely online and includes a very quick and fair peer-review system, which is all easy to use. Visit http://www.dovepress.com/testimonials.php to read real quotes from published authors. 